\title{
Comparison of dark energy models: A perspective from the latest observational data
}

Physicists at the Institute of Theoretical Physics, Chinese Academy of Sciences and the Department of Physics at Northeastern University have made a comparison of a number of competing dark energy models. They have tested and compared nine popular dark energy models using the latest observational data. The study was reported in Issue 9 (Volume 53) of SCIENCE CHINA Physics, Mechanics \& Astronomy because of its significant research value.

Over the past decade, cosmologists around the world have accumulated conclusive evidence for the fact that the cosmic expansion is accelerating. Within the framework of the standard cosmological model, this implies that about two-thirds of the cosmos is composed of an exotic component, "dark energy", which, unlike any known form of matter or energy, is gravitationally repulsive. To explain the gravitationally repulsive dark energy, physicists and cosmologists have proposed a variety of theoretical models. However, none of them are commonly accepted as the convincing theoretical explanation for dark energy. The preferred candidate for dark energy is the famous cosmological constant (CC) that was originally introduced by Albert Einstein in 1917 in his static universe model (the first modern cosmological model based on general relativity) and was later called by Einstein the "biggest blunder". Among all the dark energy models, the CC model is the simplest one and can fit the observational data well, though it always suffers from severe theoretical puzzles such as the "fine-tuning" and "cosmic coincidence" problems. Understanding the nature of dark energy continues to be one of the major missions for fundamental physics.

In the absence of clear theoretical guidance, the physics community is reliant on comparison to observational data in selecting a correct dark energy model. In this work, nine popular dark energy models are tested and compared using the latest observational data, which includes type Ia supernovae, baryon acoustic oscillation, and cosmic microwave background. The models under consideration are the cosmological constantmodel, two equation of state parameterization models, the generalized Chaplygin gas model, two Dvali-Gabadadze-Porrati models, and three holographic dark energy models. All of these models are well-known dark energy candidates and have attracted considerable attention in the past.

The dark energy models have been fitted to the observational data. In this procedure, each model is assessed with a number called the information criterion IC value. Statistically, a model with fewer parameters and with a better fit to the data has a lower IC value. The models under consideration have been compared and ranked according to their IC values.

Consequently, it has been found that the CC model fits the observational data best. In addition to the CC model, five other models also provide a good fit to the current data, while the remaining three models, a Dvali-Gabadadze-Porrati model and two holographic models, clearly do not fit the observational data well (for simplicity, we call them the five good models and the three bad models). It is interesting to note that four of the five good models are closely related to the $\mathrm{CC}$ model, which may be the reason they fit the data so well, while none of the bad models can be reduced to the CC model.

Furthermore, it is interesting to note that there is one holographic dark energy model, which is not reducible to the CC model, yet it still provides a good fit to the current observational data. This model was proposed by Professor LI Miao, one of the authors of this work. As the name implies, this holographic dark energy model arises from the holographic principle of quantum gravity. The holographic principle determines the range of validity for a local effective quantum field theory to be an accurate description of the world involving dark energy by imposing a relationship between the ultraviolet and infrared cut-offs. In his article, "A Model of Holographic Dark Energy", Li has shown that a form of holographic dark energy emerges if the future event horizon size of the universe is chosen as the infrared cut-off scale in the effective quantum field theory.

In summary, the CC model is still the best candidate for dark energy, according to the results from fitting models to current observational data. The authors concluded that "Given the current quality of the observational data, and with the assumption of a flat universe, information criteria indicate that the cosmological constant model is still the best one and there is no reason to prefer any more complex model". However, as new observations are made, it is possible that the preferred model could change. It is important to keep in mind that there are limitations associated with the current observational data. To precisely determine the nature of dark energy, an improvement in the accuracy of data is of essential importance. "We look forward to seeing whether this conclusion 'that the CC model is the best candidate for dark energy' can be changed by future more accurate data," said the authors in the final statement of the paper.

This work was supported by the National Natural Science Foundation of China (10705041, 10821504, 10975032 and 10975172), and the National Basic Research Program of China (2007CB815401).

See the article: Li M, Li X D , Zhang X. Comparison of dark energy models: A perspective from the latest observational data. Sci China Phys Mech Astron, 2010, 53: 1631-1645 